\title{
Data Mining und wissenschaftliche Forschung - de lege lata und de lege ferenda
}

\author{
Melanie Graf / Kirsten Johanna Schmidt *
}

Der Vorentwurf zum neuen Urheberrechtsgesetz enthält mit Art. 24d E-URG eine Bestimmung, welche die Verwendung von Werken zu wissenschaftlichen Zwecken regelt. So soll die Vervielfältigung und Bearbeitung von urheberrechtlich geschützten Werken künftig zulässig sein, sofern dies durch die Anwendung eines technischen Verfahrens bedingt ist. Die neue Bestimmung zielt auf Fälle des so genannten Text and Data Mining $a b$, d.h. der computergestützten Suche, Analyse und Vernetzung von Daten mit dem Ziel, neue Erkenntnisse und Zusammenhänge zu erhalten. Es stellt sich die Frage, ob die in Art. 24d E-URG genannten Werknutzungen nicht bereits nach geltendem Recht zulässig sind. Der vorliegende Beitrag beschreibt zunächst den Vorgang des Text und Data Mining (I.) und dessen urheberrechtliche Relevanz (II.), bevor die möglicherweise einschlägigen Schranken des Urheberrechts untersucht werden (III.). Schliesslich wird auf die neue Bestimmung Art. 24d E-URG eingegangen (IV.).

I. Text and Data Mining (TDM) ............................................................. 186

II. Urheberrechtliche Relevanz ..................................................................... 187

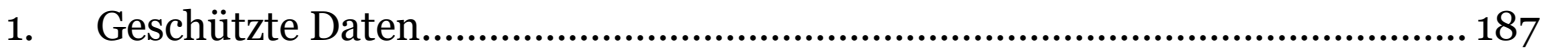

2. Urheberrechtlich relevante Vorgänge des Text und Data Mining ................. 188

III. Schranken des Urheberrechts und Data Mining ............................................191

1. Schranke der vorübergehenden Vervielfältigung (Art. 24a URG) ..................191

2. Eigengebrauchsschranke nach geltendem Recht ...................................... 194

IV. Die Wissenschaftsschranke de lege ferenda (Art. 24d E-URG)......................... 196

1. Vervielfältigung und Bearbeitung erfolgen zum Zweck der wissenschaftlichen Forschung

2. Durch ein technisches Verfahren bedingte Vervielfältigung und Bearbeitung.... 198

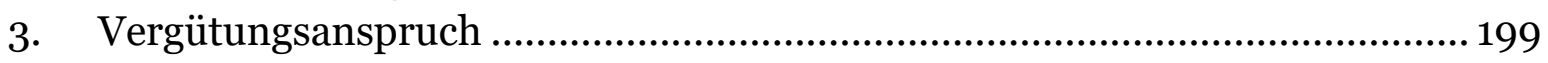

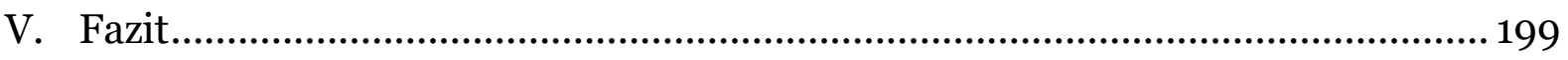

Zitiervorschlag: Melanie Graf / Kirsten Johanna Schmidt, Data Mining und wissenschaftliche Forschung - de lege lata und de lege ferenda, in: sui-generis 2017, S. 185

URL: $\quad$ sui-generis.ch/42

DOI: $\quad$ https://doi.org/10.21257/sg.42

* Melanie Graf, MLaw, und Kirsten Johanna Schmidt, MLaw, Rechtsanwältin, LL.M. (Boston), sind beide wissenschaftliche Assistentinnen der Professur für Life Sciences-Recht und Immaterialgüterrecht an der Juristischen Fakultät der Universität Basel.

Dieses Werk ist lizenziert unter einer Creative Commons Namensnennung - Weitergabe unter gleichen Bedingungen 4.0 International Lizenz. 


\section{Text and Data Mining (TDM)}

1 Data Mining dient der Gewinnung neuer Erkenntnisse, Muster und Zusammenhänge durch die Untersuchung, Analyse und Vernetzung grosser Datenmengen. ${ }^{1}$ Die Informationsverarbeitung und -gewinnung ist automatisiert und computergestützt, wodurch auch sehr grosse Datensets (Big Data) «bezwungen» werden können. ${ }^{2}$ In der biomedizinischen Forschung ermöglicht Data Mining beispielsweise, Zusammenhänge zwischen bestimmten Gensequenzen und Krankheitsbildern zu erkennen.3 Gegenstand des Data Mining können alle möglichen Arten von Daten sein, beispielsweise Videos, Bilder und Texte. 4 Beim Text Mining, einer Unterkategorie des Data Mining, werden grosse Sammlungen von digitalisierten Textdokumenten durchsucht.5

2 Text- und Data Mining-Software kann technisch sehr unterschiedlich ausgestaltet sein. Für die folgende juristische Analyse wird das TDM in drei - durch Com-

Erläuternder Bericht zu zwei Abkommen der Weltorganisation für geistiges Eigentum und zu Änderungen des Urheberrechtsgesetzes vom 11.12.2015, S. 22 f. (Bericht URG); Brändli, Data Mining als Forschungsmethode: Die Probleme des Grabens nach Datengold, in: Brändli/ Harasgama/Schister/Tamò, Mensch und Maschine - Symbiose oder Parasitismus?, Bern 2015, S. 41 ff., S. 42.

2 Brändli, (Fn. 1), S. 44 f.; Spindler, Text und Data Mining - urheber- und datenschutzrechtliche Fragen, GRUR 2016, S. 1112 ff., S. 1113; Triaille/de Meeûs d'Argenteuil/de Francquen, Study on the legal framework of text and data mining (TDM), March 2014, S. 17 mit einer detaillierten Definition von TDM; Bericht URG, (Fn. 1), S. $22 \mathrm{f}$.

3 Brändli, (Fn. 1), S. 42.

4 Brändli, (Fn. 1), S. 44 f.; Triaille et al, (Fn. 2), S. 8.

5 Brändli, (Fn. 1), S. 44 f.; Truyens/Van Eecke, Legal aspects of text mining, Computer Law and Security Review 2014, 153 ff., 153 f. puterprogramme ausgeführte - Datenverarbeitungsschritte aufgeteilt 6 : Im ersten Schritt wird mittels Suchprogrammen in zugänglichen digitalen Daten nach relevanten Daten für die konkrete Analyse gesucht.7 Die Suchergebnisse werden in einem zweiten Schritt in einen durch die Analyse-Software verarbeitbaren Datensatz umgewandelt. ${ }^{8}$ Im dritten Schritt wird die Analyse-Software auf den Datensatz angewendet, welche das Analyseresultat ausgibt.

3 Von den drei Datenverarbeitungsschritten (Suche - Transformation - Analyse) sind die verarbeiteten Daten zu unterscheiden: Zugängliche Daten, Suchergebnisse mit den relevanten Daten, verarbeitbarer Datensatz und das Analyseresultat. Die Suchergebnisse mit den relevanten Daten und der verarbeitbare Datensatz sind Output eines Datenverarbeitungsschrittes und Input für den darauffolgenden Datenverarbeitungsschritt; sie stellen Zwischenergebnisse im gesamten TDM-Prozess dar, wobei das Analyseresultat das Endergebnis ist.

4 Überblick:

$6 \overline{\text { Andere Autoren nehmen abweichende Abgren- }}$ zungen vor: z.B. Brändli, (Fn. 1), S. 44 f., m.w.N.

7 Z.B. mittels sog. Crawling- und ScrapingSoftware: Brändli, (Fn. 1), S. 45; Truyens/Van Eecke, (Fn. 5), S. 165 beschreibt Crawling als automatisiertes Durchsuchen von Webseiten und Scraping als automatisiertes Downloaden wesentlicher Bestandteile von Webseiten.

8 Z.B. in ein einheitliches Format usw. Vgl. Bericht URG, (Fn. 1), S. 23. 
3. Verarbeitung durch Analyse-Software

D. Output: Ergebnis der Datenanalyse

Zuletzt: Verwendung der Ergebnisse (Vertrieb, Anwendung der semantischen Information)

5 Bei der Verarbeitung von Daten durch Computerprogramme entstehen immer vorübergehende Vervielfältigungen der verarbeiteten Daten (Dateninput). ${ }^{9}$ Das Ergebnis der jeweiligen Datenverarbeitung (Datenoutput) wird je nach Ausgestaltung des TDM-Verfahrens permanent $^{10}$ oder aber nur vorübergehend ${ }^{11}$ auf einem Datenträger gespeichert. Eine Ausnahme stellt das Analyseergebnis dar, welches in der Regel zur weiteren Verwendung permanent gespeichert wird.

\section{Urheberrechtliche Relevanz}

\section{Geschützte Daten}

6 Die vom Data Mining betroffenen Daten können u.a. Rohdaten, semantische Daten, Texte, Bilder, Videos und Datenbanken umfassen. Während Rohdaten an sich kaum urheberrechtlich geschützt sind, ${ }^{12}$ können insbesondere gerade bei Bildern und Texten urheberrechtlich geschützte Werke vorliegen, d.h. geistige Schöpfungen der Literatur und Kunst mit individuellem Charakter gemäss Art. 2

9 Beispielsweise wird das Datenset bei der Verarbeitung durch die Analyse-Software vorübergehend in den Arbeitsspeicher (RAM) kopiert, vgl. z.B. Brändli, (Fn. 1), S. 45.

1o Durch Crawling und Scraping gewonnene Daten werden in der Regel dauernd auf einem Computer oder Server gespeichert, Brändli, (Fn. 1), S. 45; Triaille et al., (Fn. 2), S. 46.

11 Z.B. durch bloss temporäre Ablage der Daten im Arbeitsspeicher (RAM), welche nach einiger Zeit durch Überschreiben gelöscht werden, vgl. Brändli, (Fn. 1), S. 45.

12 Bericht URG, (Fn. 1), S. 23.
Abs. 1 URG. ${ }^{13}$ Da der Gesetzgeber den Bereich der Literatur und Kunst jedoch sehr weit versteht, fallen auch Werke mit wissenschaftlichem oder technischem Inhalt darunter. ${ }^{14}$ Gemäss Art. 2 Abs. 3 URG gelten auch Computerprogramme als Werke. Der Inhalt eines Werks als solcher - also der semantische Gehalt eines Werks - ist urheberrechtlich nicht geschützt, wohl aber die Art und Weise, wie dieser Inhalt mitgeteilt wird. ${ }^{15}$

7 Datenbanken sind im Schweizer Recht im Gegensatz etwa zum europäischen Recht ${ }^{16}$ - nur schutzfähig, wenn sie ein Sammelwerk im Sinne des Art. 4 Abs. 1 URG darstellen. ${ }^{17}$ Dies ist der Fall, wenn die Datenbank als Sammlung bezüglich Auswahl und Anordnung eine geistige Schöpfung mit individuellem Charakter darstellt. Sammlungen von Tatsachen ohne individuellen Charakter sind dagegen nicht geschützt. ${ }^{18}$

8 Rohdaten wie z.B. Messwerte und Nutzerdaten sind urheberrechtlich nicht geschützt. Bei personenbezogenen Daten muss jedoch insbesondere für die Sammlung, Bearbeitung und Veröffentlichung das geltende Datenschutzrecht beachtet

$13 \overline{\mathrm{Zu} \text { den einzelnen Schutzvoraussetzungen siehe }}$ z.B. von Büren/Marbach/Ducrey, Immaterialgüter- und Wettbewerbsrecht, 3. Aufl., Bern 2008, Rn. $230 \mathrm{ff}$.

14 Von Büren/Marbach/Ducrey, (Fn. 13), Rn. 236.

15 Von Büren/Marbach/Ducrey, (Fn. 13), Rn. 241. Eine Urheberrechtsverletzung liegt gemäss bundesgerichtlicher Rechtsprechung vor, wenn ein Werk in seinen charakteristischen Grundzügen hinsichtlich Planung, Auswahl und Erfassen des Stoffes oder bezüglich der Anordnung und Gliederung übernommen wird, siehe BGE 113 II 306, 64 II 165.

16 Siehe zum Datenbankherstellerschutz im Kontext von Big Data z.B. Schmidt/Zech, Datenbankherstellerschutz für Rohdaten?, CR 2017, $417 \mathrm{ff}$.

17 Von Büren/Marbach/Ducrey, (Fn. 13), Rn. 273 ff.

18 Brändli, (Fn. 1), S. 47 m.H. auf BGE 113 II 306, 308. 
werden. Davon ist vor allem die medizinische und psychologische Forschung betroffen, während in anderen Wissenschaftsdisziplinen personenbezogene Daten eher selten vorkommen.19 Auf diese Thematik soll im Rahmen dieses Beitrags nicht näher eingegangen werden. ${ }^{20}$

\section{Urheberrechtlich relevante Vorgänge des Text und Data Mining}

\section{a) Permanente Speicherung der verarbeiteten Datenwerke}

9 Eine urheberrechtlich relevante Vervielfältigung liegt vor, sobald das Werk in dessen wesentlichen individuellen Zügen vervielfältigt wird..$^{21}$ Bei der permanenten Speicherung der Suchergebnisse mit den relevanten Daten oder zumindest deren individuellen Elemente liegt klar eine durch menschliche Sinne wahrnehmbare Vervielfältigung i.S.v. Art. 10 Abs. 2 lit. a URG vor. ${ }^{22}$ Bei der Zusammenführung dieser Werke oder Werkteile kann ein Sammelwerk nach Art. 4 URG entstehen. ${ }^{23}$ Werden nur nicht individuelle Elemente der zugänglichen Datenwerke gespeichert, liegt keine urheberrechtlich relevante Vervielfältigung vor.

Werden die Suchergebnisse für die spätere Verarbeitung durch Analyse-Software

$19 \overline{\text { Die Autorinnen und Autoren wissenschaftlicher }}$ Werke willigen mit der Publikation in die Bearbeitung (Art. 3 lit. e DSG) ihrer Namen ein.

20 Dazu bspw. Schweizer, Data mining, data warehousing: datenschutzrechtliche Orientierungshilfen für Privatunternehmen, Zürich 1999.

21 Vgl. Rehbinder/Viganò, OFK URG, 3. Aufl., Zürich 2008, URG $10 \mathrm{Rn} .1$.

22 Brändli, (Fn. 1), S. 50; vgl. auch Spindler, (Fn. 2), S. 1112.

23 Vgl. auch Weber, Big Data: Rechtliche Perspektive, in: Weber/Thouvenin, Big Data und Datenschutz - Gegenseitige Herausforderungen, S. 17 ff., S. 22. in ein anderes Format umgewandelt, strukturiert oder/und bereinigt, entsteht je nach Einzelfall ein Werk zweiter Hand nach Art. 3 URG oder aber eine blosse Vervielfältigung. ${ }^{24}$ In beiden Fällen ist eine Zustimmung des Rechteinhabers erforderlich. Enthält das Suchergebnis nur nicht individuelle Elemente der online zugänglichen Daten, handelt es sich um eine freie Benutzung. Freie Benutzungen liegen vor, sofern die neue Schöpfung die Vorlagewerke nicht mehr erkennen lässt.25 Der freien Benutzung vorangehende Vervielfältigungen der Vorlagewerke bleiben jedoch urheberrechtlich relevant.

11 Das Ergebnis der TDM-Analyse kann die relevanten Bild-, Video- oder Textstellen der analysierten Daten selbst anzeigen, was eine urheberrechtlich relevante Nutzungshandlung darstellt. In der Regel werden die Analyseergebnisse aber bloss die (semantische) Information enthalten, welche aus den analysierten Daten extrahiert wurde. Zwischen diesen beiden Möglichkeiten sind unzählige Mischformen bzw. Varianten denkbar. Je nach dem liegen Vervielfältigungen, Werke der zweiten Hand, Sammelwerke oder aber

$24 \overline{\mathrm{Ob} \text { eine Vervielfältigung oder ein Werk zweiter }}$ Hand vorliegt, hängt davon ab, wie sehr das veränderte Werk vom ursprünglichen Werk abweicht. Vgl. Barrelet/Egloff, Das neue Urheberrecht, 3. Aufl., Bern 2008, URG 3 Rn. 4; Rehbinder/Viganò, (Fn. 21), URG 3 Rn. 1. Zwischen Art. 11 Abs. 1 lit. a URG bzw. lit. b und Art. 10 Abs. 2 lit. a URG besteht keine Normenkonkurrenz, beide können kumulativ verletzt werden: Hilty, Urheberrecht, Bern 2011, Rn. 156. Gegen das Vorliegen einer urheberrechtlichen Bearbeitung nach $\S 23$ UrhG bei der Erstellung eines verarbeitbaren Datensatzes: Spindler, GRUR 2016, 1112, 1113. Denkbar ist jedoch auch eine sog. «freie Benutzung».

25 Vgl. statt aller Rehbinder/Viganò, (Fn. 21), URG 3 Rn. 1. 
eine freie Benutzung vor. Dies muss jedoch im Einzelfall beurteilt werden.

\section{b) Vorübergehende Speicherung der einzelnen Datenwerke}

Während der Verarbeitung der Datenwerke durch Computerprogramme entstehen immer Vervielfältigungen der Dateninputs im RAM, welche unter Art. 24a URG fallen können. ${ }^{26}$ Davon zu unterscheiden ist die bloss vorübergehende Speicherung der Zwischenergebnisse (Suchergebnis und verarbeitbarer Datensatz). Es ist denkbar, dass die Zwischenergebnisse direkt durch den darauffolgenden Datenverarbeitungsprozess weiterverarbeitet werden, so dass zwischen den zugänglichen Datenwerken und dem Analyseergebnis keine permanenten Speicherungen erfolgen. Hier stellt sich die Frage, ob überhaupt eine urheberrechtlich relevante Werkverwendung oder bloss reiner Werkgenuss bzw. eine freie Benutzung vorliegt.

Urheberrechtlich relevante Nutzungshandlungen knüpfen an die Vorgänge der Werkvermittlung an, der reine Werkgenuss, als Wahrnehmung mittels menschlicher Sinne, ist dagegen frei. ${ }^{27}$ Beispielsweise ist das Lesen eines urheberrechtlich geschützten Buches keine urheberrechtlich relevante Nutzungshandlung; das Lesen als Wahrnehmung durch menschliche Sinne gilt als reiner Werkgenuss, da dieser keine weiteren Vorgänge der Werkvermittlung erfordert. ${ }^{28}$

26 Dazu sogleich.

27 Hilty, (Fn. 24), Rn. 150.

28 Vgl. Barrelet/Egloff, (Fn. 24), URG 10 Rn. 6a; Rehbinder/Viganò, (Fn. 21), URG 10 Rn. 4. dingt nicht möglich. Die Wahrnehmung digitaler Werke durch menschliche Sinne setzt deren - wenn auch nur vorübergehende - Vervielfältigung voraus. ${ }^{29}$ So wird beispielsweise beim Browsen eine temporäre Kopie der Webseite im Arbeitsspeicher für deren Darstellung auf dem Bildschirm erstellt. Das Lesen der Bildschirmdarstellung durch Menschen ist auch hier Werkgenuss, dieser erfordert im Gegensatz zum Werkgenuss analoger Werke vorübergehende Vervielfältigungen. Dieser technisch bedingte Vorgang der Werkvermittlung schliesst reinen Werkgenuss aus. Vervielfältigungen, welche durch elektronische Datenverarbeitung entstehen und der Werkvermittlung an die menschlichen Sinne dienen, gelten nach herrschender Lehre immer als urheberrechtlich relevante Vervielfältigungen nach Art. 10 Abs. 2 lit. a URG. 30 Man könnte aber auch argumentieren, dass das jeweilige Werk bei der Suchund Analysefunktion von TDM-Software - vergleichbar mit der Wahrnehmung durch menschliche Sinne - durch den Computer bloss «gelesen» wird, weshalb dieser Vorgang dem reinen Werkgenuss gleichzustellen sei. ${ }^{31}$ Dies gilt noch mehr, da die Vervielfältigungen durch TDMSoftware nicht der Werkvermittlung an die menschlichen Sinne, sondern allein der Datenverarbeitung bzw. computergestützten Analyse dienen.

$29 \overline{\text { Vgl. Egloff, Wissenschaftliche Forschung und }}$ Urheberrecht - Zur Notwendigkeit eines Forschungsprivilegs, Medialex 2009, S. 11 ff., S. 11.

30 Vgl. Barrelet/Egloff, (Fn. 24), URG 10 Rn. 12 m.w.H (herrschende Meinung). So auch Rehbinder/Viganò, (Fn. 21), URG 10 Rn. 8. Diese herrschende Meinung bezieht sich in entsprechenden Beispielen ausschliesslich auf Vervielfältigungen welche bei elektronischen Datenverarbeitungen mit dem Zweck der Werkvermittlung zum anschliessenden Werkgenuss durch menschliche Sinne entstehen.

${ }_{31}$ So Brändli, (Fn. 1), S. 8. 
Erfolgen beim gesamten TDM-Prozess keine permanenten Speicherungen der Zwischenergebnisse und stellt das Analyseergebnis eine unabhängige Schöpfung dar, stellt sich auch die Frage, ob die dabei entstehenden vorübergehenden Vervielfältigungen überhaupt Vervielfältigungen i.S.v. Art. 10 Abs. 2 lit. a URG darstellen. Urheberrechtlich relevante Vervielfältigungen dienen der mittelbaren oder unmittelbaren Wahrnehmung des vervielfältigten Werks durch menschliche Sinne. Zumindest ermöglichen solche die Wahrnehmung des Werks in irgendeiner Weise. ${ }^{2}$ Anders als beim Browsen, Streamen, Downloaden oder beim Caching dienen die Vervielfältigungen im Rahmen von TDM nicht der Wahrnehmung durch menschliche Sinne, sondern ausschliesslich der Verarbeitung der Daten durch Computerprogramme zur Gewinnung neuer Erkenntnisse.33 Werden also keine permanenten Suchergebnisse oder Datensätze erstellt, werden alleine die Analyseergebnisse mit menschlichen Sinnen wahrgenommen. Häuptli spricht von einem «one use input», weil das zu analysierende Werk einmalig in den Arbeitsspeicher des Computers geladen werden muss, während das Ergebnis der Analysesoftware keine Vervielfältigung des Werkes dar-

$32 \overline{\text { Rehbinder/Viganò, (Fn. 21), URG } 10 \text { Rn. 7: Ver- }}$ vielfältigungen dienen der unmittelbaren oder mittelbaren Wahrnehmung der Werks durch menschliche Sinne oder ermöglichen die Wahrnehmung in irgendeiner Weise, wie z.B. durch Wiedergabe; Hilty, (Fn. 24), Rn. 156: Vervielfältigung i.S. Art. 10 Abs. 2 lit. a URG liegt nur vor, sofern diese die Wahrnehmung des Werks erlaubt. So auch die deutsche Lehre, vgl. Loewenheim, in: Schricker/Loewenheim, Urheberrecht, 5. Aufl., München 2017, § 16 UrhG Rn. 5.

33 «TDM [zielt] nicht auf ein Lesen der Werke und damit nicht auf einen Werkgenuss ab, sondern auf eine Verarbeitung der darin enthaltenen Daten», Bericht URG, (Fn. 1), S. 92. stellt.34 Weil das Analyseprogramm in der Regel nicht auf die Vervielfältigung im Arbeitsspeicher zugreifen kann, wird das Vorliegen einer Vervielfältigung im urheberrechtlichen Sinne verneint. 35

16 Einerseits scheint das Abstellen auf die Werkvermittlung zur Wahrnehmung durch die menschlichen Sinne im Zeitalter der Informationstechnologie als zu eng, andererseits kann die vorübergehende Vervielfältigung im Arbeitsspeicher als mittelbar wahrnehmbar betrachtet werden, solange diese irgendwie wahrnehmbar gemacht werden könnten. ${ }^{6}$ So gelten denn auch Vervielfältigungen eines Computerprogramms, welche beim Laufenlassen desselben entstehen, gem. Art. 17 Abs. 1 lit. a URV als Werkexemplare, was auch für andere Werke gelten sollte. ${ }^{37}$ Schliesslich wäre es auch möglich von einer eigenen (neuen) Nutzungsart zu sprechen. Eine neue Nutzungsart liegt vor, sobald diese nach Verkehrsauffassung «als solche hinreichend klar abgrenzbar ist und wirtschaftlichtechnisch als einheitlich und selbständig erscheint». 38

\section{c) Konkludente Einwilligung}

17 Umstritten ist auch, ob eine konkludente Einwilligung des Rechteinhabers zur Durchführung von Data Mining durch das Zugänglichmachen der Werke im In-

34 Häuptli, Vorübergehende Vervielfältigungen im schweizerischen, europäischen und amerikanischen Urheberrecht, Diss. Basel 2004, S. 193.

35 Häuptli, (Fn. 34), S. 194.

36 A.A. Häuptli, (Fn. 34), S. 72 f., welcher argumentiert, das Werk müsse «aus sich selbst heraus» wahrnehmbar gemacht werden können. Die Wahrnehmbarmachung durch ein zweites Computerprogramm genüge dafür nicht.

37 Vgl. Loewenheim, (Fn. 32), § 16 UrhG Rn. 17.

38 Rehbinder/Viganò, (Fn. 21), URG 10 Rn. 2, in Anlehnung an das deutsche Recht. 
ternet angenommen werden kann. Eine konkludente Einwilligung wird beispielsweise angenommen für Vervielfältigungen, die durch SuchmaschinenCrawler, welche die gefundenen Daten auf einem Server abspeichern, hergestellt werden, wenn keine Willensäusserung gegen die Erfassung durch Suchmaschinen vorliegt. Die konkludente Einwilligung wird in diesem Fall darin gesehen, dass der Betrieb frei zugänglicher Webseiten dem Ziel grösstmöglicher Erreichbarkeit dient, wozu die Indexierung durch Suchmaschinen gehöre.39 Aus demselben Grund muss eine konkludente Einwilligung für das Erstellen vorübergehender Vervielfältigungen von Werken auf frei zugänglichen Webseiten für die Darstellung im Browser angenommen werden. $4^{0}$ In beiden Fällen basiert die konkludente Einwilligung auf dem Ziel der Wahrnehmung der online zugänglichen Werke.41 Da es bei TDM gerade nicht um die Wahrnehmung sondern um die computergestützte Analyse der Werke geht, ist eine konkludente Einwilligung zur Erstellung dafür erforderlicher Vervielfältigungen in Form von Zwischenergebnissen abzulehnen. ${ }^{42}$

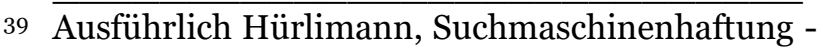
Zivilrechtliche Verantwortlichkeit der Betreiber von Internetsuchmaschinen aus Urheber-, Marken-, Lauterkeits-, Kartell- und Persönlichkeitsrecht, Bern 2012, S. 67 f.

$40 \mathrm{Bu}$, Die Schranken des Urheberrechts im Internet, Bern 2004, S. 157 m.w.N. Inzwischen wurde für diesen und ähnliche Fälle die Schranke der vorübergehenden Vervielfältigung (Art. 24a URG) eingeführt, welche jedoch ebenfalls eine rechtmässige Nutzung des Vorlagewerks voraussetzt, die in der konkludenten Einwilligung oder im freien Werkgenuss gesehen werden kann, dazu unten.

41 Siehe dazu auch die Urteile Vorschaubilder I und II des deutschen BGH (BGH vom 29.4.2010, Az. 1 ZR 69/o8, und BGH vom 19.10.2011, Az. 1 ZR 140/10).

42 A.A. Weber, (Fn. 23), S. 22; Zieger/Smirra, MMR 2013, 418, 419, jedoch keine konkludente

\section{d) Fazit}

18 Vervielfältigungen individueller Elemente von Datenwerken bedürfen einer Lizenz des Rechteinhabers; das gilt für die permanente Speicherung der Suchergebnisse, des Datensatzes und des Analyseergebnisses sowie die nur vorübergehende Speicherung der Zwischenergebnisse in einem «one use input»Verfahren. Auch vorübergehende Vervielfältigungen während der Datenverarbeitung gelten als Vervielfältigungen, also die vorübergehende Vervielfältigung der durchsuchten Datenwerke während der Suche, der Suchergebnisse während der Transformation und des verarbeitbaren Datensatzes während der Analyse. Das Analyseergebnis kann und wird i.d.R. eine freie Benutzung darstellen, d.h. das Analyseergebnis darf vertrieben werden, weil es sich dabei um eine unabhängige Schöpfung handelt. Vorangehende Vervielfältigungen bedürfen auch in diesem Fall einer Einwilligung des Rechteinhabers.

19 Eine konkludente Einwilligung in Vervielfältigungen zur Verwendung in einem TDM-Verfahren liegt nicht vor. Allerdings kommt die Eigengebrauchsschranke nach Art. 19 URG und für vorübergehende Vervielfältigung die Schranke des Art. $24 a$ URG in Betracht.

\section{Schranken des Urheberrechts und Data Mining}

1. Schranke der vorübergehenden Vervielfältigung (Art. 24a URG)

20 Durch TDM-Software entstehende Vervielfältigungen könnten unter die Schran-

Einwilligung für die Vervielfältigung eines ganzen Datenbankwerks, 420. 
ke der vorübergehenden Vervielfältigung fallen. Flüchtige oder begleitende Vervielfältigungen von Werken sind gem. Art. 24a URG zulässig, sofern sie kumulativ43 einen integralen und wesentlichen Teil eines technischen Verfahrens darstellen, ausschliesslich der Übertragung der Werke zwischen Dritten durch einen Vermittler oder einer rechtmässigen Nutzung dienen und keine eigene wirtschaftliche Bedeutung haben. Unter Art. $24 a$ URG fallen beispielsweise Vervielfältigungen durch Browsing und Caching, sofern sie den Voraussetzungen entsprechen. 44

\section{a) Flüchtige oder begleitende Vervielfältigung}

21 Vorübergehende Vervielfältigungen sind nicht dauerhafte Speicherungen von Werken. Sie müssen flüchtig, d.h. besonders kurzlebige Vervielfältigungen sein, oder im Rahmen des technischen Verfahrens begleitend entstehen. 45

Vervielfältigungen, welche während der Verarbeitung mit TDM-Computerprogrammen entstehen, qualifizieren als flüchtige Vervielfältigungen. Nach Beendigung des Verfahrens werden die Vervielfältigungen im RAM mit anderen vorübergehenden Vervielfältigungen überschrieben. ${ }^{6}$ Die Speicherung der Zwischenergebnisse im Rahmen eines «one use input»-Verfahrens qualifizieren ebenfalls als vorübergehend, sofern sie nach Beendigung des TDM-Verfahrens

Botschaft zum Bundesbeschluss über die Genehmigung von zwei Abkommen der Weltorganisation für geistiges Eigentum und zur Änderung des Urheberrechtsgesetzes vom 10. März 2006 (BBl 2006), $3430 \mathrm{f}$.

44 BBl 2006, (Fn. 43), 3431.

45 Rehbinder/Viganò, (Fn. 21), URG 24a Rn. 4.

46 Brändli, (Fn. 1), S. 51 f. nicht mehr verfügbar sind bzw. mit anderen Daten überschrieben werden. Nicht als vorübergehende Vervielfältigung qualifiziert die dauerhafte Speicherung von Datenwerken. 47

\section{b) Integraler und wesentlicher Bestandteil eines technischen Verfahrens}

23 Die vorübergehenden Vervielfältigungen müssen integraler und wesentlicher Bestandteil eines technischen Verfahrens sein. Integrale und wesentliche vorübergehende Vervielfältigungen sind für das Funktionieren des technischen Verfahrens unumgänglich 48 und dienen ausschliesslich dem technischen Verfahren49. Das Ergebnis des technischen Verfahrens stellt keine integrale und wesentliche Vervielfältigung dar. ${ }^{50}$

24 Computerprogramme stellen technische Verfahren im Sinne des Art. 24a URG dar. Während der Datenverarbeitung durch Computerprogramme entstehende Vervielfältigungen sind technisch zwingend und dienen ausschliesslich der Datenverarbeitung. Das gilt für Vervielfältigungen der durchsuchten Daten während der Suche, Vervielfältigungen der Suchergebnisse während der Transformation und Vervielfältigungen des verarbeitbaren Datensatzes während der Analyse durch Computerprogramme.

25 Suchergebnis und Datensatz stellen Zwischenergebnisse eines «one use input»-

\footnotetext{
$4 7 \longdiv { \text { So auch Spindler, (Fn. 2), S. 1115. Die durch TDM } }$ angefertigten Werkkopien können gem. Spindler kaum als nur zeitweilig und erst recht nicht nur als der Übermittlung dienend angesehen werden.

48 Barrelet/Egloff, (Fn. 24), URG 24a Rn. 5.

49 Rehbinder/Viganò, (Fn. 21), URG 24a Rn. 6; Oertli, SHK URG, URG 24a Rn. 8.

5o Oertli, (Fn. 49), URG 24a Rn. 8.
} 
TDM-Verfahrens dar und damit kein integraler und wesentlicher Bestandteil. Inund Outputs sind für das Funktionieren von Computerprogrammen nicht unumgänglich, sie sind austauschbar und dienen nicht der Datenverarbeitung, sie werden verarbeitet bzw. sind das Ergebnis der Verarbeitung.

\section{c) Ausschliesslich der Übertra- gung in einem Netz zwischen Drit- ten durch einen Vermittler oder einer rechtmässigen Nutzung die- nend}

Durch TDM entstehende Vervielfältigungen dienen i.d.R. nicht der Übertragung in einem Netz zwischen Dritten durch einen Vermittler, ${ }^{51}$ sie können aber einer rechtmässigen Nutzung dienen. Eine rechtmässige Nutzung liegt vor, wenn die Nutzung der Vervielfältigungsvorlage durch eine Lizenz oder Schranke urheberrechtlich erlaubt ist. $5^{2}$

Vorübergehende Vervielfältigungen der Inputs während der elektronischen Datenverarbeitung dienen der rechtmässigen Nutzung der Datenwerke, sofern eine Schranke (z.B. Art. 19 URG) greift oder eine ausdrückliche Einwilligung des Rechteinhabers für Vervielfältigungen der Datenwerke vorliegt.53 Dasselbe gilt für die vorübergehende Speicherung der Zwischenergebnisse (d.h. der Suchergebnisse und des Datensatzes) im Rahmen

51 Spindler, (Fn. 2), S. 1115.

52 Vgl. Rehbinder/Viganò, (Fn. 21), URG 24a Rn. 8; Oertli, (Fn. 49), URG 24a Rn. 11.

53 Vorübergehende Vervielfältigungen von rechtmässig erstellten dauerhaften Datensätzen, welche bei der Analyse durch Software entstehen, dienen der Nutzung des legal erstellten Datensatzes und dienen der rechtmässigen Nutzung derselben, Oertli, (Fn. 49), URG 24a Rn. 11; vgl. auch Zieger/Smirra, MMR 2013, 418, 419. eines «one use input»-TDM-Verfahrens. 54

28 Stellt das Analyseergebnis eine unabhängige Schöpfung dar, liegt eine freie Benutzung vor. Wird ein solches durch ein «one use input»-Verfahren hergestellt, dienen die vorangehenden Vervielfältigungen einer rechtmässigen Nutzung.

\section{d) Keine eigene wirtschaftliche Bedeutung der vorübergehenden Vervielfältigungen}

29 Eröffnen vorübergehende Vervielfältigungen andere oder intensivere, 55 neue oder eigenständige56 Nutzungsmöglichkeiten, kommt den vorübergehenden Vervielfältigungen eine eigene wirtschaftliche Bedeutung $\mathrm{zu}$, welche die Anwendung der Schranke ausschliesst. An einer eigenständigen wirtschaftlichen Bedeutung fehlt es, wenn die vorübergehenden Vervielfältigungen die eigentliche Werknutzung nur begleiten.57 Beim Browsing liegt die eigentliche Werknutzung in der Wahrnehmung durch Menschen.

3o Vorübergehende Vervielfältigungen der Inputs während der Datenverarbeitung durch Computerprogramme dienen allein dem technischen Verfahren und haben deshalb keine eigenständige wirtschaftliche Bedeutung. ${ }^{8}$ Die eigentliche

$54 \overline{\text { Vgl. zur rechtmässigen Nutzung von Datenwer- }}$ ken oben, Kap. II 2. Urheberrechtlich relevante Vorgänge und Kap. III 2. zur Schranke des Eigengebrauchs.

55 Barrelet/Egloff, (Fn. 24), URG 24a Rn. 7. Oertli, (Fn. 49), URG 24a Rn. 12 spricht von qualitativ und quantitativ anderer Nutzung der Werkexemplare.

56 Rehbinder/Viganò, (Fn. 21), URG 24a Rn. 9.

57 Vgl. BBl 2006, (Fn. 43), 3430.

58 So im Ergebnis auch Brändli, (Fn. 1), S. 52, bezüglich vorübergehender Vervielfältigungen welche während der Verarbeitung von rechtmässig erstellten Datensätze durch Analyse-Software entstehen. 
Werknutzung liegt in der Suche, Transformation oder Analyse von Daten durch Computerprogramme, wobei die vorübergehenden Vervielfältigungen bloss begleitend auftreten.

31 Die Zwischenergebnisse in einem «one use input»-Verfahren erhalten hingegen eine eigene wirtschaftliche Bedeutung, weil diese Vervielfältigungen keine blosse Hilfsfunktion erfüllen.59 Für das Vorliegen eigener wirtschaftlicher Bedeutung der Zwischenergebnisse spricht auch, dass dadurch Kosten für Vervielfältigungsexemplare der Daten vermieden werden. ${ }^{60}$

\section{e) Fazit}

Die während der Datenverarbeitung entstehenden vorübergehenden Vervielfältigungen der Inputs fallen bei rechtmässiger Nutzung der Vorlagewerke unter Art. 24a URG. Die vorübergehende Speicherung der Zwischenergebnisse fällt dagegen nicht unter Art. 24a URG. Hier scheitert die Anwendung der Schranke am Fehlen der Voraussetzung des integralen und wesentlichen Bestandteils eines technischen Verfahrens, sowie am Vorliegen einer eigenen wirtschaftlichen Bedeutung der vorübergehenden Vervielfältigung.

\section{Eigengebrauchsschranke nach geltendem Recht}

\section{a) Werkverwendung im persönlichen Bereich}

33 Gemäss Art. 19 Abs. 1 lit. a URG dürfen veröffentlichte Werke ${ }^{61}$ im persönlichen

\footnotetext{
59 Zieger/Smirra, (Fn. 53), 418, 419.

6o Vgl. Barrelet/Egloff, (Fn. 24), URG 24a Rn. 7.

61 Mit Ausnahme von Computerprogrammen, Art. 19 Abs. 4 URG.
}

Bereich und im Kreis von unter sich eng verbundenen Personen, wie Verwandten oder Freunden, ohne Zustimmung des Rechteinhabers verwendet werden.

34 Der Privatgebrauch ist natürlichen Personen vorbehalten ${ }^{62}$, dies lässt sich nach der grammatikalischen Auslegungsmethode schon der Formulierung der Schrankenbestimmung entnehmen. ${ }^{63}$ Die Voraussetzung der engen Verbundenheit «verlangt also mehr als eine irgendwie geartete persönliche Beziehung».64 Ausserhalb der Familie und familienähnlichen Lebensgemeinschaften bedarf es «einer dauerhaften, wirklich persönlichen freundschaftlichen Verbundenheit».65 Arbeitgeber und Arbeitnehmer sowie Mitarbeiter eines Betriebes erfüllen diese Voraussetzung grundsätzlich nicht. ${ }^{66}$ Dasselbe gilt für Vereinsmitglieder67, Mitglieder einer Partei oder einer religiösen Gemeinschaft ${ }^{68}$ sowie Bekanntschaften, welche «im Internet oder

62 Cherpillod, Schranken des Urheberrechts, in: Von Büren/David (Hrsg.), Schweizerisches Immaterialgüter- und Wettbewerbsrecht II/1, Urheberrecht und verwandte Schutzrechte, 3. Aufl., Basel 2014, Rn. 772; Barrelet/Egloff, (Fn. 24), URG 19 Rn. 8; Gasser, SHK URG, 2. Aufl., Bern 2012, URG 19 Rn. 6, wohl herrschende Meinung. Dessemontet, Le droit d'auteur, Lausanne 1999, S. 314; Gasser, Der Eigengebrauch im Urheberrecht, Bern 1997, S. 50; Brändli, (Fn. 1), S. 52.

${ }_{63}$ Cherpillod, (Fn. 62), Rn. 772; Barrelet/Egloff, (Fn. 24), URG 19 Rn. 8.

64 Barrelet/Egloff, (Fn. 24), URG 19 Rn. 8.

65 Rehbinder/Viganò, (Fn. 21), URG 19 Rn. 16; vgl. auch Gasser, Eigengebrauch, (Fn. 62), S. 56 ff. m.w.N.

66 Cherpillod, (Fn. 62), Rn. 774 m.w.N. auf kantonale Gerichtsentscheide. Cour de justice GE, SMI 1978, S. 121: Die Sendung von Musik anlässlich eines Betriebsfestes ist bewilligungspflichtig. Siehe auch OGer AG, SMI 1992 II, S. 55 (Aufführung von Musikstücken an Betriebsfeiern).

67 Cherpillod, (Fn. 62), Rn. 774 (Verweis auf OGer ZH, SJZ 64 (1968), S. 198, sog. «Privatclub». Siehe auch Cour de justice GE, SMI 1983 I, S. 107.); Barrelet/Egloff, (Fn. 24), URG 19 Rn. 8.

68 Barrelet/Egloff, (Fn. 24), URG 19 Rn. 8. 
über andere Medien im Wesentlichen zum Zweck des Austauschs von Werken begründet werden» 69 .

Betreibt also eine natürliche Person Data Mining für ein persönliches Projekt, ist dies durch die Schranke des Privatgebrauchs gerechtfertigt.70 Sobald es sich jedoch um ein Forschungsteam, eine Kooperation o.ä. handelt, was zweifelsohne den praktisch relevanteren Fall darstellt, greift diese Schranke nicht mehr. ${ }^{71}$

\section{b) Betriebsinterner Gebrauch}

36 Allenfalls könnte in diesem Fall die Schranke des betriebsinternen Gebrauchs gemäss Art. 19 Abs. 1 lit. c URG Anwendung finden. Danach dürfen veröffentlichte Werke für die interne Information oder Dokumentation in Betrieben, öffentlichen Verwaltungen, Instituten, Kommissionen oder ähnlichen Einrichtungen vervielfältigt werden. Die zur Vervielfältigung berechtigten Einheiten sind sehr weit gefasst und es fallen sowohl private als auch öffentliche Betriebe in den Geltungsbereich der Schranke.72 Somit könnten Forschungseinrichtungen und -gruppen sich auf die Schranke des betriebsinternen Gebrauchs berufen, aber nur so lange, als keine betriebsfremden Personen miteinbezogen werden. 73

Neben der blossen Vervielfältigung wird auch das Recht von dieser Schranke erfasst, die Vervielfältigungsexemplare betriebsintern zu verbreiten und elektro-

69 Rehbinder/Viganò, (Fn. 21), URG 19 Rn. 16.

Brändli, (Fn. 1), S. 53.

Bericht URG, (Fn. 1), S. 23.

Cherpillod, (Fn. 62), Rn. 814.

Egloff, (Fn. 29), S. 16; Bericht URG, (Fn. 1), S. 23. nisch zugänglich zu machen. ${ }^{74}$ Die Vervielfältigung von Werken in analoger und in digitaler Form ist zulässig.75 Umformatierungen von Werken in andere Formate sind dementsprechend möglich, nicht aber sonstige Veränderungen bzw. Bearbeitungen. ${ }^{76}$ Je nach Verarbeitung der Werke durch die TDM-Software das jeweilige Werk wird bloss vervielfältigt oder auch verändert - kann der Anwendungsbereich dieser Schranke bei TDM-Fällen bereits an dieser Stelle ausgeschlossen werden. Gemäss Art. 19 Abs. 3 lit. a URG wird ausserdem die (weitgehend) vollständige Vervielfältigung von Werken nicht von der Schranke erfasst, 77 wobei gemäss Art. 19 Abs. $3^{\text {bis }}$ URG Vervielfältigungen, die beim Abrufen von erlaubterweise zugänglich gemachten Werken hergestellt werden, von dieser Einschränkung des Eigengebrauchs ausgenommen sind. 78

38 Anlass zu Diskussionen im Falle des Data Mining gibt die Zweckbestimmung der Vervielfältigung für die interne Information oder Dokumentation. Die Vervielfältigung zu Dokumentationszwecken ist die Sammlung, systematische Erfassung und Ordnung von Wissensträgern, deren Aufbewahrung der späteren Information

74 Egloff, (Fn. 29), S. 15, m.H. auf BGE 133 III 540; Gasser, Eigengebrauch, (Fn. 62), S. 110 f.; Cherpillod, (Fn. 62), Rn. 811.

75 Hilty, (Fn. 24), Rn. 226: «technologieneutral».

76 Gasser, SHK URG, (Fn. 62), URG 19 Rn. 20; Hilty, (Fn. 24), Rn. 225; Rehbinder/Viganò, (Fn. 21), URG 19 Rn. 26; vgl. Bericht URG, (Fn. 1), S. 23.

77 Bericht URG, (Fn. 1), S. 23.

78 So im Ergebnis auch Brändli, (Fn. 1), S. 54 f., welche in Erwägung zieht, die Schranke mittels teleologischer Auslegung auf vollständige Vervielfältigung von Werken im RAM während der Analyse durch TDM-Software anzuwenden. Permanente Kopien bei der Erstellung eines Datensatzes liessen sich hingegen nur schwerlich unter die Schranke subsumieren. 
der Mitglieder des Betriebs dient.79 Die Vervielfältigung zum Zweck der internen Information betrifft die Vermittlung von Wissen (semantischer Information) an die Mitglieder der jeweiligen Einrichtung; 80 Ziel ist also die Wahrnehmung durch die Mitglieder des Betriebs. Erfasst werden gemäss Bundesgericht sogar Vervielfältigungen, welche vor allem der Arbeitserleichterung und weniger der Wissensvermittlung dienen. ${ }^{81}$ Folge der Zweckbeschränkung auf interne Information und Dokumentation ist, dass sich der Inhalt der Vervielfältigungen auf den Zweck der vervielfältigenden Einrichtung bezieht. ${ }^{82}$ Diese Zweckbestimmung der betriebsinternen Nutzung von Werken schliesst die klassische wissenschaftliche Forschung vermutlich nicht aus. ${ }^{83}$ Die Vervielfältigung von Daten zur Analyse durch Software dient aber nicht nur der Vermittlung von Wissen an Mitglieder der jeweiligen Einrichtung, sondern vor allem der Verarbeitung durch Software und der anschliessenden Verwertung der Ergebnisse. Dagegen können die durch TDM entstehenden Kopien durchaus (auch) der internen Information und Dokumentation dienen; dies gilt insbesondere für das erstellte Datenset. ${ }^{84} \mathrm{Ob}$ der eigentliche Text und Data MiningVorgang tatsächlich nur der internen Information und Dokumentation dient, erscheint zweifelhaft und hat soweit er-

79 Gasser, Eigengebrauch, (Fn. 62), S. 94. m.w.N.

80 Gasser, Eigengebrauch, (Fn. 62), S. 94.

81 BGE 108 II 475 («Pressespiegel PTT»). Als Beispiel wird die Kopie einschlägiger Seiten eines (zuvor erworbenen) Kommentars in einer Anwaltskanzlei angeführt, BGE 108 II 457, $481 \mathrm{f}$.

82 Gasser, Eigengebrauch, (Fn. 62), S. 95.

83 Hilty, (Fn. 24), Rn. 226. In Forschungseinrichtungen läuft die Nutzung von Werken für die Forschung auf - technologieneutral zu verstehende Vervielfältigungen zum Zwecke der Information oder Dokumentation hinaus.

84 Brändli, (Fn. 1), S. 55. sichtlich noch keine gerichtliche Beurteilung erfahren.

\section{c) Fazit}

39 Als Ergebnis kann festgehalten werden, dass - je nach Ausgestaltung der Software - einige TDM-Vorgänge nicht von der Eigengebrauchsschranke erfasst werden. ${ }^{85}$ Insbesondere beim betriebsinternen Gebrauch stellen sich zudem vergütungsrechtliche Fragen, da der Nutzer dem Urheber für die gesetzliche Lizenz eine Vergütung schuldet. 86 Wirklich ungehindert kann mit urheberrechtlich geschützten Werken also de lege lata nicht geforscht werden. ${ }^{87}$

40 Die Publikation der Forschungsergebnisse fällt im Übrigen nicht unter die Eigengebrauchsschranke. ${ }^{88}$ Hierbei sind - neben den Standards zum wissenschaftlichen Arbeiten - allenfalls die Bestimmungen zu Werken zweiter Hand (Art. 3 URG) sowie zu Zitaten (Art. 25 URG) zu beachten.

\section{Die Wissenschaftsschranke de lege ferenda (Art. 24d E-URG)}

41 Text und Data Mining fallen in den Anwendungsbereich der neuen in Art. 24d E-URG enthaltenen Wissenschaftsschranke, welche die Verwendung urheberrechtlich geschützter Werke für die wissenschaftliche Forschung (teilweise) erlauben soll. ${ }^{89}$ Urheberrechtliche Schrankenregelungen müssen den internationalen Anforderungen des Drei-Stufen-Tests nach Art. 9 Abs. 2 RBÜ, Art. 13 TRIPS

\footnotetext{
85 A.A. Brändli, (Fn. 1), S. 55; Egloff, (Fn. 29), S. 16.

86 Art. 20 Abs. 2 URG; Brändli, (Fn. 1), S. 55.

87 Egloff, (Fn. 29), S. 17.

88 Egloff, (Fn. 29), S. 16.

89 Bericht URG, (Fn. 1), S. 61.
} 
und Art. 10 WCT entsprechen.90 Der enge Tatbestand und vorgesehene Vergütungsanspruch der neuen Wissenschaftsschranke beeinträchtigen im Ergebnis die normale Verwertung der Originalwerke und die Interessen der Urheber nicht in unzumutbarer Weise, weil die Schranke nur das Vervielfältigungs- und das Bearbeitungsrecht betrifft, sofern diese durch ein technisches Verfahren bedingt sind, und auf wissenschaftliche Forschung beschränkt ist. 91

\section{Vervielfältigung und Bearbeitung erfolgen zum Zweck der wissen- schaftlichen Forschung}

Die Wissenschaftsschranke setzt zunächst voraus, dass Vervielfältigungen und Bearbeitungen zum Zweck der wissenschaftlichen Forschung erfolgen (Abs. 1). Wissenschaftliche Forschung ist die systematische Suche nach neuen Erkenntnissen innerhalb einer oder mehrerer wissenschaftlicher Disziplinen, wie z.B. den Naturwissenschaften, den Ingenieurswissenschaften und den Geisteswissenschaften. ${ }^{2}$ Kritisch ist in diesem Zusammenhang anzumerken, dass nach dieser sehr weiten Definition quasi jede Tätigkeit als Forschung qualifiziert, solange nur systematisch nach neuen Erkenntnissen gesucht wird. Eine Eingrenzung wäre über das Kriterium der Wissenschaftlichkeit möglich, zum Beispiel indem eine Forschung gefordert wird, welche den wissenschaftlichen Prinzipien und Regeln der jeweiligen Disziplin entspricht bzw. auf anerkannten wissenschaftlichen Standards beruht.

\footnotetext{
Vgl. Hilty, (Fn. 24), Rn. 215.

Bericht URG, (Fn. 1), S. 62.

Bericht URG, (Fn. 1), S. 61.
}

43 Neben der systematischen Suche nach neuen Erkenntnissen können Forschungstätigkeiten auch noch anderen Zwecken dienen.93 Im Anwendungsbereich der Wissenschaftsschranke ist es gemäss des Erläuternden Berichts zur URG Änderung nicht erforderlich, dass die Forschung ausschliesslich einen wissenschaftlichen Zweck verfolgt; die wissenschaftliche Forschung soll aber Hauptzweck der Arbeiten bleiben.94 Unseres Erachtens genügt für die Anwendung der Wissenschaftsschranke, dass das Kriterium der wissenschaftlichen Forschung erfüllt ist. Weitere Zwecke können nur darin gesehen werden, wozu die Ergebnisse dieser Forschung verwendet werden. Ein Abwägen von Hauptzwecken und möglichen Nebenzwecken ist abzulehnen: Eine solche Abwägung ist erstens nicht praktikabel, zumal hier auch subjektive Komponenten eine Rolle spielen können. Zweitens sieht der Wortlaut der Norm Art. 24d E-URG eine solche Abwägung nicht vor. Dementsprechend ist nicht $\mathrm{zu}$ berücksichtigen, $\mathrm{zu}$ welchen Zwecken die Forschungsergebnisse verwendet werden sollen. In diesem Sinne unterscheidet Art. 24d E-URG auch nicht zwischen Forschung zu kommerziellen und nicht kommerziellen Zwecken.95 Die Unterscheidung wurde als impraktikabel erachtet, weil verschiedene Finanzierungsformen und For-

93 Bericht URG, (Fn. 1), S. 62.

94 Bericht URG, (Fn. 1), S. 61.

95 Im Gegensatz etwa zu Art. 5 Abs. 3 Bst. a InfoSocRL (Richtlinie 2001/29/EG des Europäischen Parlaments und des Rates v. 22.5.2001 zur Harmonisierung bestimmter Aspekte des Urheberrechts und der verwandten Schutzrechte in der Informationsgesellschaft, ABl. L 167, 22.6.2001, 10 ff.) oder Art. 6 Abs. 2 Bst. b Datenbank-RL (Richtlinie 96/9/EG des Europäischen Parlaments und des Rates v. 11.3.1996 über den rechtlichen Schutz von Datenbanken, ABl. L 77, 27.3.1996, $20 \mathrm{ff}$.). 
schungstätigkeiten oftmals nicht nur einem einzigen Zweck dienen. So bestehen z.B. bei staatlich finanzierten Forschungsprojekten an Hochschulen, welche kommerziell verwertbare Ergebnisse liefern, oder bei durch Privatwirtschaftsunternehmen finanzierten Forschungsprojekten von Non-profit-organisationen Unklarheiten. ${ }^{96}$

\section{Durch ein technisches Verfahren bedingte Vervielfältigung und Bearbeitung}

44 Die Vervielfältigungen und Bearbeitungen müssen durch ein technisches Verfahren zur wissenschaftlichen Forschung bedingt sein. 97 Das heisst, die Vervielfältigungen oder Bearbeitungen müssen im Rahmen des technischen Verfahrens anfallen oder erfolgen, um das technische Verfahren anwenden zu können. ${ }^{8}$ Es ist jedoch noch unklar, welche technischen Verfahren genau erfasst werden sollen, zumal heute ausserordentlich viele Vorgänge technisiert sind. Der Zugang zu den genutzten Werken muss allerdings rechtmässig sein; 99 d.h. die Werke müssen entweder rechtmässig erworben oder z.B. im Internet frei zugänglich sein.

45 Vervielfältigungen und Bearbeitungen, welche im Rahmen des Text und Data Mining anfallen, werden von Art. 24d EURG erfasst. Im Gegensatz zu Art. 24a URG werden hier auch permanente Vervielfältigungen erfasst. ${ }^{100}$ Ein erstellter und bearbeiteter Datensatz darf dauerhaft gespeichert werden, auch die Ver-

96 Bericht URG, (Fn. 1), S. 62.

97 Bericht URG, (Fn. 1), S. 61.

98 Bericht URG, (Fn. 1), S. 61, vgl. auch die vom Wortlaut her eindeutigere französische Fassung, S. 60 .

99 Bericht URG, (Fn. 1), S. 92.

100 Bericht URG, (Fn. 1), S. 61. vielfältigung und Bearbeitung ganzer Werke ist zulässig. ${ }^{101}$ Es ist dagegen nicht erlaubt, Vervielfältigungen zu erstellen, welche nur dazu dienen sollen, Kosten für den Erwerb weiterer Exemplare des urheberrechtlich geschützten Werkes einzusparen. ${ }^{102}$ Ausserdem darf das Forschungsergebnis das Originalwerk nicht ersetzen. ${ }^{103}$ Dies wird in der Forschung insbesondere bei Text und Data Mining wohl nur selten der Fall sein, da eine grosse Anzahl von Werken verwendet wird. ${ }^{104}$

46 Erlaubt ist in Anwendung der Wissenschaftsschranke einzig die Vervielfältigung und Bearbeitung der Werke. ${ }^{105}$ Ein erstellter Datensatz darf also nicht vertrieben oder der Öffentlichkeit zugänglich gemacht werden. Die aus der Forschung resultierenden Ergebnisse dürfen vertrieben werden, sofern sie als von den verwendeten Originalwerken als unabhängig anzusehen sind. Stellt das Forschungsresultat lediglich ein Werk zweiter Hand oder ein Sammelwerk dar, bedarf der Vertrieb der Einwilligung sämtlicher Rechteinhaber der Originalwerke.

47 Computerprogramme dürfen gem. Art. 24d Abs. 4 E-URG nicht für Forschungszwecke vervielfältigt und bearbeitet werden, sie sind von der Schranke ausgenommen. Begründet wird dies damit, dass die Entwicklung von Computerprogrammen komplex, aufwändig und mit Investitionen verbunden sei. ${ }^{106}$ 


\section{Vergütungsanspruch}

48 Der Vorentwurf enthält einen Vergütungsanspruch der Rechteinhaber der Originalwerke für die Vervielfältigung und Bearbeitung ihrer Werke. Dieser kann jedoch nur über eine Verwertungsgesellschaft geltend gemacht werden.107 In der Vernehmlassung wurde die Vergütung teilweise abgelehnt. ${ }^{108}$ Auch die Arbeitsgruppe zum Urheberrecht des IGE empfahl schliesslich eine vergütungsfreie Wissenschaftsschranke. ${ }^{109}$ Das Eidgenössische Justiz- und Polizeidepartement EJPD wird voraussichtlich bis Ende 2017 dem Bundesrat eine Botschaft zur Revision des URG unterbreiten. ${ }^{110} \mathrm{Ob}$ die Wissenschaftsschranke vergütungsfrei sein wird oder nicht, steht also noch nicht endgültig fest.

\section{Fazit}

49 Die Einführung einer Wissenschaftsschranke ist grundsätzlich zu begrüssen. ${ }^{111}$ Zwar können für den Bereich des «eigentlichen» Data Mining, d.h. der Datenanalyse, die Voraussetzungen der Schranke für vorübergehende Vervielfältigungen gemäss Art. 24a URG erfüllt sein. ${ }^{112}$ Allerdings werden nicht alle nötigen Schritte des gesamten Prozesses, wie in diesem Beitrag dargestellt, von dieser

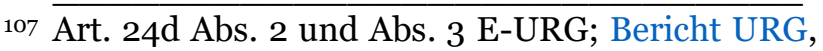
(Fn. 1), S. 62.

108 Eidgenössisches Institut für Geistiges Eigentum IGE, Zwei Abkommen der Weltorganisation für geistiges Eigentum und Änderungen des Urheberrechtsgesetzes, Bericht über das Ergebnis des Vernehmlassungsverfahrens, vom 2. Dezember 2016, S. 12.

109 Medienmitteilung der Arbeitsgruppe zum Urheberrecht vom 2. März 2017, S. 2.

110 Webpage des EJPD, Modernisierung des Urheberrechts.

111 Ebenso Egloff, (Fn. 29), S. 18, welcher zudem auf die Erhöhung der Attraktivität der Schweiz als Forschungsplatz hinweist.

112 Brändli, (Fn. 1), S. 59. urheberrechtlichen Schranke erfasst. Beispielsweise ist die Erstellung permanenter Datensets bisher nur sehr eingeschränkt für die Forschung möglich. ${ }^{113}$

50 Private Lizenzlösungen erscheinen gerade bei Big Data Sachverhalten, wo immense Mengen an Daten verarbeitet werden, nicht praktikabel. Zudem werden TDM-Verfahren in den AGB verschiedener Verlage ausgeschlossen. ${ }^{114}$ Es ist in der Literatur umstritten, ob insbesondere die Eigengebrauchsschranke vertraglich ausschliessbar oder dagegen zwingender Natur ist. Das Bundesgericht erklärte die urheberrechtlichen Schranken ohne nähere Begründung als zwingend. ${ }^{115}$ Teile der Lehre dagegen argumentieren für die vertragliche Abbedingbarkeit.116 Die Einführung einer Wissenschaftsschranke ist u.E. nur sinnvoll, wenn diese Schranke als zwingend ange-

${ }_{113} \overline{\text { Vgl. Brändli, (Fn. 1), S. 60, wonach aber die ge- }}$ naue Ausgestaltung einer Schranke, welche das Erstellen von grossen Datensets zulässt, «auf jeden Fall sorgfältig durchdacht werden» sollte.

114 Bericht URG, (Fn. 1), S. 24, wo das Einholen von Lizenzen bei den Rechteinhabern als «kostenintensive Barriere der Forschungstätigkeit» bezeichnet wird.

115 BGE 127 III 26; zustimmend Barrelet/Egloff, (Fn. 24), URG 19 Rn. 2a; Egloff, Urheberrechtsabgaben für digitale Speichergeräte Bundesgerichtsentscheid vom 19. Juni 2007 (2A.53/2006, 2A.322/2006, 2A.336/2006, 2A.337/2006, 2A.338/2006), Medialex 2007, S. 136 ff., 146. Mit Hinweis auf die in C457/11 C460/11 vom 27. Juni 2013 zum Ausdruck gebrachte gleichlautende Auffassung des Europäischen Gerichtshofs Egloff, Zur Vergütungspflicht für private Fotokopien, sic! 2014, S. 165 ff., 168; wohl vorsichtig zustimmend Wigger/Handle, EuGH: Neues und weniger Neues zur Privatkopievergütung - insbesondere zu ihrem Verhältnis $\mathrm{zu}$ individuell vereinbarten Vergütungen und $\mathrm{zu}$ technischen Schutzmassnahmen, sic! 2015, S. 464 ff., 472.

116 Cherpillod, (Fn. 62), Rn. 750; Hilty, (Fn. 24), Rn. 216; Rehbinder/Viganò, (Fn. 21), URG 19 Rn. 7. Für einen Überblick siehe z.B. Wigger/ Handle, (Fn. 115), 472 ff. 
sehen wird. Mit Einführung der Wissenschaftsschranke könnte auch die Frage, «ob das vertraglich eingeräumte Recht zur Nutzung der Inhalte einer Datenbank auch das Recht beinhaltet, die Inhalte zum Zwecke der Forschung mithilfe von Data Mining-Software zu analysieren»117, zumindest für die wissenschaftliche Forschung offengelassen werden.

51 Auch für die Rechtssicherheit bei der wissenschaftlichen Forschung ist die Einführung einer Wissenschaftsschranke förderlich, zumal bei genauerer Analyse der neuen technischen Möglichkeiten nicht einfach $\mathrm{zu}$ beantwortende (urheber-)rechtliche Fragen aufgeworfen werden. ${ }^{118}$ 